\title{
On the stability of a class of splitting methods for integro-differential equations
}

\author{
A. Araújo ${ }^{\mathrm{a}, *}$, J.R. Branco ${ }^{\mathrm{b}}$, J.A. Ferreira ${ }^{\mathrm{a}}$ \\ ${ }^{\text {a }}$ CMUC, Department of Mathematics, University of Coimbra, Apartado 3008, 3000 Coimbra, Portugal \\ ${ }^{\mathrm{b}}$ Department of Physics and Mathematics, Insituto Superior de Engenharia de Coimbra, \\ Rua Pedro Nunes-Quinta da Nora, 3030-199 Coimbra, Portugal
}

\begin{abstract}
The classical convection-diffusion-reaction equation has the unphysical property that if a sudden change in the dependent variable is made at any point, it will be felt instantly everywhere. This phenomena violate the principle of causality. Over the years, several authors have proposed modifications in an effort to overcome the propagation speed defect. The purpose of this paper is to study, from analytical and numerical point of view a modification to the classical model that take into account the memory effects. Besides the finite speed of propagation, we establish an energy estimate to the exact solution. We also present a numerical method which has the same qualitative property of the exact solution. Finally we illustrate the theoretical results with some numerical simulations.
\end{abstract}

(c) 2008 IMACS. Published by Elsevier B.V. All rights reserved.

Keywords: Integro-differential equations; Splitting methods; Stability; Convergence

\section{Introduction}

The classical heat equation for the temperature $u$

$$
\frac{\partial u}{\partial t}(x, t)=\frac{k}{\gamma} \frac{\partial^{2} u}{\partial x^{2}}(x, t),
$$

on a bar, where $\gamma$ represents the heat capacity, is obtained combining the Fourier's law for the heat flux $q$

$$
q(x, t)=-k \frac{\partial u}{\partial x}(x, t),
$$

where $k$ denotes the thermal conductivity, with the Mass Conservation law

$$
\frac{\partial u}{\partial t}(x, t)+\frac{\partial q}{\partial x}(x, t)=0 .
$$

\footnotetext{
* Corresponding author.

E-mail addresses: alma@mat.uc.pt (A. Araújo), jrbranco@isec.pt (J.R. Branco), ferreira@ mat.uc.pt (J.A. Ferreira).

URLs: http://www.mat.uc.pt/ alma (A. Araújo), http://www.mat.uc.pt/ ferreira (J.A. Ferreira).
} 
The classical heat equation has the unphysical property that if a sudden change in the temperature is made at a point of the bar, it will be felt instantly everywhere. This property, known as a infinite speed of propagation, is not present in heat conduction phenomena and is consequence of the violation of principle of causality by the Fourier law for the flux. In fact, this law establishes that the observed heat flux at some point at some time is consequence of the space temperature variation at the same point and at the same time.

In order to overcome the limitation of the traditional heat equation, over the years, several authors have proposed modifications to Fourier's flux including in its definition a certain memory term as an effort to avoid the infinite propagation speed $[5,12,11]$.

Attending that the heat flux $q$ at point $x$ and at time $t$ should be consequence of the temperature variation at point $x$ but at some passed time, Cattaneo, in [5], proposed the following heat flux definition

$$
q(x, t+\tau)=-k \frac{\partial u}{\partial x}(x, t),
$$

where $\tau$ is a relaxation time. Considering a first-order approximation to the flux and integrating the first-order differential equation

$$
\frac{\partial q}{\partial t}(x, t)+\frac{1}{\tau} q(x, t)=-\frac{k}{\tau} \frac{\partial u}{\partial x}(x, t),
$$

we obtain the so-called Cattaneo's flux

$$
q(x, t)=-\frac{k}{\tau} \int_{0}^{t} e^{-\frac{t-s}{\tau}} \frac{\partial u}{\partial x}(x, s) \mathrm{d} s .
$$

Note that, when $\tau \rightarrow 0$, the Cattaneo's flux tends to the classical Fourier's flux. Combining (2) with (1) we obtain, for the temperature, the Cattaneo's equation

$$
\frac{\partial u}{\partial t}(x, t)=\frac{k}{\tau \gamma} \int_{0}^{t} e^{-\frac{t-s}{\tau}} \frac{\partial^{2} u}{\partial x^{2}}(x, s) \mathrm{d} s .
$$

The simplest initial boundary value problem (IBVP) - (3) with $u(x, 0)=u_{0}(x)$ - that gives rise to finite speed of propagation is defined using (3) [5,12]. In fact, if we impose some regularity on the initial condition $u_{0}$, we may prove that this IBVP is equivalent to a hyperbolic IBVP defined by telegraph equation

$$
\frac{\partial^{2} u}{\partial t^{2}}(x, t)+\frac{1}{\tau} \frac{\partial u}{\partial t}(x, t)=\frac{k}{\gamma \tau} \frac{\partial^{2} u}{\partial x^{2}}(x, t),
$$

that transmits waves with finite velocity $c=\sqrt{k /(\gamma \tau)}$.

In [11], Joseph and Preziosi argue that there is no real conductor where the heat conduction phenomenon can be modeled by the Cattaneo's equation. So, they propose the use of a modified flux defined by

$$
q(x, t+\tau)=-k \frac{\partial u}{\partial x}\left(x, t+\tau^{*}\right), \quad \tau>\tau^{*},
$$

with two relaxation parameters. Considering the first-order approximation to the flux and to the gradient of the concentration we obtain

$$
\frac{\partial q}{\partial t}(x, t)+\frac{1}{\tau} q(x, t)=-\frac{k}{\tau} \frac{\partial u}{\partial x}(x, t)-k \frac{\tau^{*}}{\tau} \frac{\partial}{\partial t} \frac{\partial u}{\partial x}(x, t),
$$

which allows us to obtain the following heat flux

$$
q(x, t)=-k \frac{\tau^{*}}{\tau} \frac{\partial u}{\partial x}(x, t)-\frac{k}{\tau}\left(1-\frac{\tau^{*}}{\tau}\right) \int_{0}^{t} e^{-\frac{t-s}{\tau}} \frac{\partial u}{\partial x}(x, s) \mathrm{d} s .
$$


If we take $k=k_{1}+k_{2}$, where $k_{1}$ represent the effective thermal conductivity and $k_{2}$ the elastic conductivity, and $\tau^{*} / \tau=k_{1} / k$, we obtain the so-called Jeffrey's heat flux [11]

$$
q(x, t)=-k_{1} \frac{\partial u}{\partial x}(x, t)-\frac{k_{2}}{\tau} \int_{0}^{t} e^{-\frac{t-s}{\tau}} \frac{\partial u}{\partial x}(x, s) \mathrm{d} s .
$$

From the Mass Conservation law (1) it is easy to show that, in this case, the temperature $u$ satisfies the Jeffrey's equation

$$
\frac{\partial u}{\partial t}(x, t)=\frac{k_{1}}{\gamma} \frac{\partial^{2} u}{\partial x^{2}}(x, t)+\frac{k_{2}}{\tau \gamma} \int_{0}^{t} e^{-\frac{t-s}{\tau}} \frac{\partial^{2} u}{\partial x^{2}}(x, s) \mathrm{d} s .
$$

In the last years several analytical and numerical studies on the solution of IBVP defined by using integrodifferential equation as the Jeffrey's equation, arise in the literature. For instance, in [2], the authors considered the Jeffrey's equation

$$
\frac{\partial u}{\partial t}(x, t)=\alpha \frac{\partial^{2} u}{\partial x^{2}}(x, t)+\frac{D}{\tau} \int_{0}^{t} e^{-\frac{t-s}{\tau}} \frac{\partial^{2} u}{\partial x^{2}}(x, s) \mathrm{d} s, \quad x \in(a, b), t>0,
$$

with homogeneous Dirichlet boundary conditions. From an analytical point of view they establish an energy estimate which was fundamental to prove the stability of the IBVP with respect to perturbations of the initial condition. From a numerical viewpoint they propose a splitting method which simulates the heat transport as the superposition of two phenomena: diffusion and memory in time, being the memory treated by using the telegraph equation.

Reaction-diffusion integro-differential equations have been also considered in the literature in order to overcome some unphysical behavior presented by the solution of the classical Fisher equation

$$
\frac{\partial u}{\partial t}(x, t)=D \frac{\partial^{2} u}{\partial x^{2}}(x, t)+f(u(x, t)) .
$$

In fact, if the reaction term $f$ is defined by $f(u(x, t))=U u(x, t)(1-u(x, t))$, then the traveling wave solution $u(x, t)=\phi(x-c t)$ connecting the stationary states $u=0$ (unstable) and $u=1$ (stable) satisfies $c \geqslant \sqrt{4 D U}$. Then when the reaction parameter $U$ goes to infinity, the propagation speed $c$ goes also to infinity and this behavior is unphysical [6,7]. For instance, in [4], the Fisher-Kolmogorov-Petrovskii-Piskunov equation

$$
\frac{\partial u}{\partial t}(x, t)=f(u(x, t))+\frac{D}{\tau} \int_{0}^{t} e^{-\frac{t-s}{\tau}} \frac{\partial^{2} u}{\partial x^{2}}(x, s) \mathrm{d} s, \quad x \in(a, b), t>0,
$$

with homogeneous Dirichlet boundary conditions, was studied from analytical and numerical point of view. The stability of the model was established and some numerical methods were proposed.

In [8] and [9], reaction-transport systems with memory and long range interaction were modeled by the following integro-differential equation

$$
\frac{\partial u}{\partial t}(x, t)=\int_{0}^{t} \alpha(t-s)\left(\int_{\mathbb{R}} u(x+\mu, s) \phi(\mu) d \mu-u(x, s)\right) \mathrm{d} s+f(u(x, t)), \quad x \in \mathbb{R},
$$

where $\alpha(s)$ and $\phi(\mu)$ represent kernel functions. The initial value problem defined by (8) was studied in [10] from analytical and numerical point of view where estimates for the $L^{2}$ norm of the solution and the $L^{2}$ norm of its past were established. These estimates were deduced for the continuous model and for the discrete models proposed in that paper. 
The use of memory terms in the definition of the flux in some biological applications leads to new models. These new models enables us to study quantities that the classical models do not give any information. For instance, in [3], it was considered a new model for percutaneous absorption of a drug which consists in integro-differential equation

$$
\frac{\partial u}{\partial t}(x, t)=\mu-\gamma u(x, t)+\beta \frac{\partial u}{\partial x}(x, t)+\frac{D}{\tau} \int_{0}^{t} e^{-\frac{t-s}{\tau}} \frac{\partial^{2} u}{\partial x^{2}}(x, s) \mathrm{d} s, \quad x \in(a, b), t>0,
$$

with appropriate initial and boundary conditions. The authors studied the qualitative properties of the model and its numerical approximation and they compared their model with the classical one based on the classical Fick's law for the flux.

In this paper we will consider the IBVP

$$
\frac{\partial u}{\partial t}(x, t)=f(u(x, t))+\beta \frac{\partial u}{\partial x}(x, t)+\alpha \frac{\partial^{2} u}{\partial x^{2}}(x, t)+\frac{D}{\tau} \int_{0}^{t} e^{-\frac{t-s}{\tau}} \frac{\partial^{2} u}{\partial x^{2}}(x, s) \mathrm{d} s, \quad x \in(a, b), t>0,
$$

where $\alpha, D \geqslant 0, \tau>0, \beta \in \mathbb{R}$, with initial and boundary conditions

$$
\begin{aligned}
& u(x, 0)=u_{0}(x), \quad x \in(a, b), \\
& u(a, t)=u_{a}(t), \quad u(b, t)=u_{b}(t), t>0 .
\end{aligned}
$$

Our aim is to study the qualitative properties of the solution of (10)-(11) and the stability of the model with respect to the $L^{2}$ norm and also with respect of the $L^{2}$ norm of the past in time of the gradient. From the numerical point of view we propose splitting methods which allow us to compute numerical approximations presenting the qualitative behavior of the solution of (10)-(11). As the models considered in [1-4] are particular cases of the general model (10), the stability results for the continuous model can be seen as generalizations of the stability results presented in the mentioned papers. In what concerns the numerical point of view, a splitting method for the integro-differential model with $\beta=0$ was considered in [2] but replacing the subproblem defined by the integro-differential term by a telegraph equation. In that paper the stability results were established with respect to a discrete $L^{2}$ norm. The splitting method that we study in the present paper is based on the functional splitting considered in [2] but using the integro-differential version. Such approach enable us to characterize a discrete $L^{2}$ norm of the discrete solution as well the norm of the past in time of the space numerical derivative of such solution.

The paper is organized as follows. In Section 2 the qualitative behavior of model (10)-(11) is studied and its stability is concluded. In Section 3 we study a family of $\theta$ numerical methods in terms of its stability and accuracy. Finally, in Section 4 some numerical simulations are included illustrating the theoretical results obtained in Section 3.

\section{Energy estimates}

Let us consider the IBVP (10)-(11). We use the following notation: by $v(t)$ we denote the $x$-function if $v$ is defined in $[a, b] \times[0, T]$ and $t$ is fixed.

We establish, in the following result, an estimate for the energy functional

$$
E(u)(t)=\|u(t)\|^{2}+\frac{D}{\tau}\left\|\int_{0}^{t} e^{-\frac{t-s}{\tau}} \frac{\partial u}{\partial x}(s) \mathrm{d} s\right\|^{2},
$$

for $t>0$, where $\|\cdot\|$ represents the usual $L^{2}$ norm.

Theorem 1. Let $u$ be a solution of (10)-(11) with homogeneous boundary conditions, satisfying, for each $t \in[0, T]$, $|u(x, t)| \leqslant L\left(L \in \mathbb{R}^{+}\right)$, for $x \in[a, b]$, and $\frac{\partial u}{\partial t}(t), \frac{\partial u}{\partial x}(t), \frac{\partial^{2} u}{\partial x^{2}}(t), \int_{0}^{t} e^{-\frac{t-s}{\tau}} \frac{\partial^{2} u}{\partial x^{2}}(s) \mathrm{d} s \in L^{2}[a, b]$. If $f$ is continuously differentiable and $f(0)=0$, then

$$
E(u)(t) \leqslant e^{2 \max \left\{-\frac{1}{\tau}, f_{\max }^{\prime}-\frac{\alpha}{(b-a)^{2}},\right\} t}\left\|u_{0}\right\|^{2},
$$

for each $t \in(0, T]$, where $f_{\max }^{\prime}=\max _{|u| \leqslant L} f^{\prime}(u)$. 
Proof. Multiplying (10) by $u$, with respect to the $L^{2}$ inner product $(\cdot, \cdot)$ and integrating by parts, we easily get

$$
\frac{1}{2} \frac{d}{d t}\|u(t)\|^{2} \leqslant f_{\max }^{\prime}\|u(t)\|^{2}-\alpha\left\|\frac{\partial u}{\partial x}(t)\right\|^{2}-\frac{D}{\tau}\left(\int_{0}^{t} e^{-\frac{t-s}{\tau}} \frac{\partial u}{\partial x}(s) \mathrm{d} s, \frac{\partial u}{\partial x}(t)\right) .
$$

As

$$
\left(\int_{0}^{t} e^{-\frac{t-s}{\tau}} \frac{\partial u}{\partial x}(s) \mathrm{d} s, \frac{\partial u}{\partial x}(t)\right)=\frac{1}{2} \frac{d}{d t}\left\|\int_{0}^{t} e^{-\frac{t-s}{\tau}} \frac{\partial u}{\partial x}(s) \mathrm{d} s\right\|^{2}+\frac{1}{\tau}\left\|\int_{0}^{t} e^{-\frac{t-s}{\tau}} \frac{\partial u}{\partial x}(s) \mathrm{d} s\right\|^{2},
$$

we deduce from (14) the differential inequality

$$
\frac{d}{d t} E(u)(t) \leqslant 2 \max \left\{-\frac{1}{\tau}, f_{\max }^{\prime}-\frac{\alpha}{(b-a)^{2}}\right\} E(u)(t),
$$

which allows us to obtain (13).

According to the previous theorem, the solution $u$ satisfies

$$
\|u(t)\| \leqslant e^{\max \left\{-\frac{1}{\tau}, f_{\max }^{\prime}-\frac{\alpha}{(b-a)^{2}}\right\} t}\left\|u_{0}\right\|
$$

and the "average in time" of its gradient

$$
\left\|\int_{0}^{t} e^{-\frac{t-s}{\tau}} \frac{\partial u}{\partial x}(s) \mathrm{d} s\right\| \leqslant e^{\max \left\{-\frac{1}{\tau}, f_{\max }^{\prime}-\frac{\alpha}{(b-a)^{2}}\right\} t}\left\|u_{0}\right\| .
$$

If $f_{\max }^{\prime}<0$ then

$$
\|u(t)\| \rightarrow 0 \text { and } \quad\left\|\int_{0}^{t} e^{-\frac{t-s}{\tau}} \frac{\partial u}{\partial x}(s) \mathrm{d} s\right\| \rightarrow 0, \quad \text { as } t \rightarrow \infty .
$$

Remark 1. We remark that, as particular cases, we conclude the following:

1. For the IBVP defined by the Fisher-Kolmogorov-Petrovskii-Piskunov equation (7) we have, as in [4],

$$
E(u)(t) \leqslant e^{2 \max \left\{-\frac{1}{\tau}, f_{\max }^{\prime}\right\} t}\left\|u_{0}\right\|^{2}, \quad t \geqslant 0 .
$$

2. For the IBVP defined by the Fisher equation (6) it can be shown that

$$
\|u(t)\| \leqslant e^{f_{\max }^{\prime} t}\left\|u_{0}\right\| .
$$

3. For the Jefferey's IBVP defined by (5) holds, as in [1],

$$
E(u)(t) \leqslant e^{-2 \min \left\{\frac{1}{\tau}, \frac{\alpha}{(b-a)^{2}}\right\} t}\left\|u_{0}\right\| \rightarrow 0, \quad \text { as } t \rightarrow \infty .
$$

4. For Cattaneo's IBVP we may conclude that

$$
E(u)(t) \leqslant\left\|u_{0}\right\|^{2} \text {. }
$$

5. For the classical heat IBVP it is known that

$$
\|u(t)\| \leqslant e^{-\frac{\alpha}{(b-a)^{2}} t}\left\|u_{0}\right\| \rightarrow 0, \quad \text { as } t \rightarrow \infty .
$$

Let us now consider the stability behavior of the solution $u$ under perturbations in the initial condition $u_{0}$.

Let $u$ and $u_{\epsilon}$ be solutions of (10) satisfying the same boundary conditions and initial conditions $u_{0}$ and $u_{0}+\epsilon$, respectively. The influence of $\epsilon$ on the solution of is estimated in the following result. 
Theorem 2. Let $u$ and $u_{\epsilon}$ be solutions of (10) satisfying the same boundary conditions and initial conditions $u_{0}$ and $u_{0}+\epsilon$, respectively. If, for these solutions, the hypothesis of Theorem 1 are satisfied then

$$
E\left(u-u_{\epsilon}\right)(t) \leqslant e^{2 \max \left\{-\frac{1}{\tau}, f_{\max }^{\prime}-\frac{\alpha}{(b-a)^{2}},\right\} t}\|\epsilon\|^{2},
$$

for each $t \in(0, T]$, where $f_{\max }^{\prime}=\max _{|u| \leqslant L} f^{\prime}(u)$.

Proof. Let us first note that $v_{\epsilon}=u-u_{\epsilon}$ satisfies

$$
\begin{aligned}
\frac{\partial v_{\epsilon}}{\partial t}(x, t)= & f(u(x, t))-f\left(u_{\epsilon}(x, t)\right)+\beta \frac{\partial v_{\epsilon}}{\partial x}(x, t) \\
& +\alpha \frac{\partial^{2} v_{\epsilon}}{\partial x^{2}}(x, t)+\frac{D}{\tau} \int_{0}^{t} e^{-\frac{t-s}{\tau}} \frac{\partial v_{\epsilon}}{\partial x}(x, s) \mathrm{d} s, \quad x \in(a, b), t>0,
\end{aligned}
$$

and the conditions

$$
\begin{aligned}
& u_{\epsilon}(x, 0)=-\epsilon(x), \quad x \in(a, b), \\
& u_{\epsilon}(a, t)=u_{\epsilon}(b, t)=0, \quad t>0 .
\end{aligned}
$$

Multiplying Eq. (16) by $v_{\epsilon}$ with respect to the $L^{2}$ inner product $(\cdot, \cdot)$ we obtain

$$
\begin{aligned}
\left(\frac{\partial v_{\epsilon}}{\partial t}(t), v_{\epsilon}(t)\right)= & \left(f(u(t))-f\left(u_{\epsilon}(t)\right), v_{\epsilon}(t)\right)+\beta\left(\frac{\partial v_{\epsilon}}{\partial x}(t), v_{\epsilon}(t)\right) \\
& +\alpha\left(\frac{\partial^{2} v_{\epsilon}}{\partial x^{2}}(t), v_{\epsilon}(t)\right)+\frac{D}{\tau}\left(\int_{0}^{t} e^{-\frac{t-s}{\tau}} \frac{\partial^{2} v_{\epsilon}}{\partial x^{2}}(s) \mathrm{d} s, v_{\epsilon}(t)\right) .
\end{aligned}
$$

As $\left(f(u(t))-f\left(u_{\epsilon}(t)\right), v_{\epsilon}(t)\right) \leqslant f_{\max }^{\prime}\left\|v_{\epsilon}\right\|^{2}$, the proof is concluded following the same steps of the proof of the last theorem.

The stability result - Theorem 2 - enables us to deduce for the $L^{2}$ norm of $\left(u-u_{\epsilon}\right)$ the estimate

$$
\left\|\left(u-u_{\epsilon}\right)(t)\right\| \leqslant e^{\max \left\{-\frac{1}{\tau}, f_{\max }^{\prime}-\frac{\alpha}{(b-a)^{2}}\right\} t}\|\epsilon\| .
$$

From the same result we have for the $L^{2}$ norm of the "average in time" of the gradient of $\left(u-u_{\epsilon}\right)$ the following

$$
\left\|\int_{0}^{t} e^{-\frac{t-s}{\tau}} \frac{\partial}{\partial x}\left(u-u_{\epsilon}\right)(s) \mathrm{d} s\right\| \leqslant e^{\max \left\{-\frac{1}{\tau}, f_{\max }^{\prime}-\frac{\alpha}{(b-a)^{2}}\right\} t}\|\epsilon\| .
$$

Then if $\epsilon$ is small enough, we conclude that the two quantities

$$
\left\|\left(u-u_{\epsilon}\right)(t)\right\| \text { and }\left\|\int_{0}^{t} e^{-\frac{t-s}{\tau}} \frac{\partial}{\partial x}\left(u-u_{\epsilon}\right)(s) \mathrm{d} s\right\|
$$

remain small enough in the bounded time interval $[0, T]$. If the reaction term is such that $f_{\max }^{\prime}<0$ then the estimated quantities are less than $\|\epsilon\|$. In this case, we also conclude that in the unbounded time interval $[0,+\infty)$, we have

$$
\left\|\left(u-u_{\epsilon}\right)(t)\right\| \rightarrow 0 \quad \text { and } \quad\left\|\int_{0}^{t} e^{-\frac{t-s}{\tau}} \frac{\partial}{\partial x}\left(u-u_{\epsilon}\right)(s) \mathrm{d} s\right\| \rightarrow 0, \quad \text { as } t \rightarrow \infty,
$$

independently of the perturbation $\epsilon$. 


\section{Numerical methods}

Let us consider in $[a, b]$ a grid $G_{h}=\left\{x_{i}: i=0, \ldots, N\right\}$ with $x_{0}=a, x_{N}=b$ and $x_{i}-x_{i-1}=h, i=1, \ldots, N$. In what follows, we will consider the second-order centered finite difference operator $D_{2, h}$ and $D_{h, s(\beta)}$ which corresponds to $D_{h,-}$, the first-order backward finite difference operator when $\beta<0$, or to $D_{h,+}$, the first-order forward finite difference operator when $\beta>0$, defined by the usual way. Let us also consider the time grid $\left\{t_{n}, n=0, \ldots, M\right\}$ such that $t_{0}=0, t_{M}=T$ and $t_{n+1}-t_{n}=\Delta t$.

The class of splitting methods that we study are based on the following functional splitting

I. Reaction:

$$
\left\{\begin{array}{l}
\frac{\partial u_{1}}{\partial t}(x, t)=f\left(u_{1}(x, t)\right), \quad x \in(a, b), t \in(t, t+\Delta t], \\
u_{1}(x, t)=u(x, t), \quad x \in(a, b) .
\end{array}\right.
$$

II. Advection and diffusion:

$$
\left\{\begin{array}{l}
\frac{\partial u_{2}}{\partial t}(x, t)=\beta \frac{\partial u_{2}}{\partial x}(x, t)+\alpha \frac{\partial^{2} u_{2}}{\partial x^{2}}(x, t), \quad x \in(a, b), t \in(t, t+\Delta t], \\
u_{2}(x, t)=u_{1}(x, t+\Delta t), \quad x \in(a, b) .
\end{array}\right.
$$

III. Diffusion memory:

$$
\left\{\begin{array}{l}
\frac{\partial u_{3}}{\partial t}(x, t)=\frac{D}{\tau} \int_{0}^{t} e^{-\frac{t-s}{\tau}} \frac{\partial^{2} u_{3}}{\partial x^{2}}(x, s) \mathrm{d} s, \quad x \in(a, b), t \in(t, t+\Delta t], \\
u_{3}(x, t)=u_{2}(x, t+\Delta t), \quad x \in(a, b) .
\end{array}\right.
$$

We assume that the reaction problem, the advection-diffusion problem and the diffusion-memory problem are complemented with the Dirichlet boundary conditions prescribed in the original problem.

By SM we denote the splitting method obtained combining $\mathrm{I}_{h}, \mathrm{II}_{h}$ and $\mathrm{III}_{h}$ defined by

$\mathrm{I}_{h}$. Reaction:

$$
\left\{\begin{array}{l}
u_{1, h}^{n+1}=u_{1, h}^{n}+\Delta t\left((1-\theta) f\left(u_{1, h}^{n}\right)+\theta f\left(u_{1, h}^{n+1}\right)\right), \quad \theta \in[0,1], \\
u_{1, h}^{n}=u_{h}^{n} .
\end{array}\right.
$$

$\mathrm{II}_{h}$. Advection and diffusion:

$$
\left\{\begin{array}{l}
u_{2, h}^{n+1}=u_{2, h}^{n}+\Delta t \beta D_{h, s(\beta)} u_{2, h}^{n+1}+\Delta t \alpha D_{2, h} u_{2, h}^{n+1}, \\
u_{2, h}^{n}=u_{1, h}^{n+1} .
\end{array}\right.
$$

$\mathrm{III}_{h}$. Diffusion memory:

$$
\left\{\begin{array}{l}
u_{3, h}^{n+1}=u_{3, h}^{n}+\Delta t^{2} \frac{D}{\tau} \sum_{j=1}^{n} e^{-\frac{t_{n+1}-t_{j}}{\tau}} D_{2, h} u_{h}^{j}+\Delta t^{2} \frac{D}{\tau} D_{2, h} u_{3, h}^{n+1}, \\
u_{3, h}^{n}=u_{2, h}^{n+1}
\end{array}\right.
$$

where

$$
\begin{aligned}
& u_{i, h}^{j}\left(x_{0}\right)=u_{a}\left(t_{j}\right), \quad u_{i, h}^{j}\left(x_{N}\right)=u_{b}\left(t_{j}\right), \quad i=1,2,3, j=1, \ldots, M-1, \\
& u_{h}^{0}\left(x_{i}\right)=u_{0}\left(x_{i}\right), \quad i=1, \ldots, N .
\end{aligned}
$$

Finally we will consider $u\left(x_{i}, t_{n+1}\right) \approx u_{h}^{n+1}\left(x_{i}\right)=u_{3, h}^{n+1}\left(x_{i}\right), i=1, \ldots, N$. 
As we would like to study the influence of the implicitness and the explicitness of the discretization of the reaction term in the behavior of the splitting method presented, we only considered a $\theta$-method in the discretization of the reaction problem.

The stability and accuracy properties of the described splitting method will be compared with the correspondent properties of the non-splitting scheme (NSM)

$$
\begin{aligned}
u_{h}^{n+1}= & u_{h}^{n}+\Delta t\left((1-\theta) f\left(u_{h}^{n}\right)+\theta f\left(u_{h}^{n+1}\right)\right)+\Delta t \beta D_{h, s(\beta)} u_{h}^{n+1} \\
& +\Delta t \alpha D_{2, h} u_{h}^{n+1}+\frac{D \Delta t^{2}}{\tau} \sum_{j=1}^{n+1} e^{-\left(t_{n+1}-t_{j}\right) / \tau} D_{2, h} u_{h}^{j}, \quad \theta \in[0,1] .
\end{aligned}
$$

\subsection{Stability}

In order to study the stability of the numerical methods, let us introduce some notation. We denote by $L^{2}\left(G_{h}\right)$ the space of grid functions $v_{h}$ defined in $G_{h}$ such that $v_{h}\left(x_{0}\right)=v_{h}\left(x_{N}\right)=0$. In this space, we will consider the discrete inner product

$$
\left(v_{h}, w_{h}\right)_{h}=h \sum_{i=1}^{N-1} v_{h}\left(x_{i}\right) w_{h}\left(x_{i}\right), \quad v_{h}, w_{h} \in L^{2}\left(G_{h}\right) .
$$

We denote by $\|\cdot\|_{h}$ the norm induced by this inner product. We will introduce other notations:

$$
\begin{aligned}
& \left(v_{h}, w_{h}\right)_{h+}=h \sum_{i=1}^{N} v_{h}\left(x_{i}\right) w_{h}\left(x_{i}\right), \\
& \left\|v_{h}\right\|_{h+}=\left(h \sum_{i=1}^{N} v_{h}\left(x_{i}\right)^{2}\right)^{1 / 2},
\end{aligned}
$$

for grid functions defined on $G_{h}-\left\{x_{N}\right\}$.

We remark that holds the following discrete Friedrichs-Poincaré inequality

$$
\left\|v_{h}\right\|_{h}^{2} \leqslant(b-a)^{2}\left\|D_{h,-} v_{h}\right\|_{h+}^{2} .
$$

Our goal is to obtain an estimate for the fully discrete version of the energy (12) given by

$$
E_{h}\left(u_{h}^{n+1}\right)=\left\|u_{h}^{n+1}\right\|_{h}^{2}+\frac{D}{\tau}\left\|\Delta t \sum_{j=1}^{n} e^{-\left(t_{n+1}-t_{j}\right) / \tau} D_{h,-} u_{h}^{j}\right\|_{h+}^{2} .
$$

We will prove the following result for the SM defined by $\mathrm{I}_{h}-\mathrm{III}_{h}$ and (17).

Theorem 3. Let $u_{h}^{j}$ be a solution of the SM defined by $\mathrm{I}_{h}-\mathrm{III}_{h}$ and (17), with homogeneous boundary conditions, such that $\left|u_{h}^{j}\left(x_{i}\right)\right| \leqslant L\left(L \in \mathbb{R}^{+}\right)$, for $i=1, \ldots, N$ and $j=1, \ldots$, M. If $f$ is continuously differentiable and $f(0)=0$, then

$$
E_{h}\left(u_{h}^{n+1}\right) \leqslant(S(\Delta t, \theta))^{n+1}\left\|u_{0}\right\|_{h}^{2},
$$

for $n=0, \ldots, M-1$, where the stability factor $S(\Delta t, \theta)$ is defined by

$$
S(\Delta t, \theta)=\frac{1+\Delta t(1-\theta)}{\left(1+2 \alpha \Delta t(b-a)^{-2}\right)\left(1-\Delta t\left((1-\theta) f_{\max }^{\prime 2}+2 \theta f_{\max }^{\prime}\right)\right)}
$$

for $\theta \in[0,1]$, and $f_{\max }^{\prime}=\max _{|u| \leqslant L} f^{\prime}(u)$, provided that

$$
1-\Delta t\left((1-\theta) f_{\max }^{\prime 2}+2 \theta f_{\max }^{\prime}\right)>0 .
$$


Proof. Let us first consider $\mathrm{I}_{h}$

$$
u_{1, h}^{n+1}=u_{1, h}^{n}+\Delta t\left((1-\theta) f\left(u_{1, h}^{n}\right)+\theta f\left(u_{1, h}^{n+1}\right)\right) .
$$

Multiplying this equation by $u_{1, h}^{n+1}$, with respect to the $L^{2}$ inner product $(\cdot, \cdot)_{h}$, we get

$$
\left(u_{1, h}^{n+1}, u_{1, h}^{n+1}\right)_{h}=\left(u_{1, h}^{n}, u_{1, h}^{n+1}\right)_{h}+\Delta t\left((1-\theta)\left(f\left(u_{1, h}^{n}\right), u_{1, h}^{n+1}\right)_{h}+\theta\left(f\left(u_{1, h}^{n+1}\right), u_{1, h}^{n+1}\right)_{h}\right) .
$$

Due to the fact that $f(0)=0$ we obtain

$$
\left(1-\Delta t\left((1-\theta) f_{\max }^{\prime 2}+2 \theta f_{\max }^{\prime}\right)\right)\left\|u_{1, h}^{n+1}\right\|_{h}^{2} \leqslant 1+\Delta t(1-\theta)\left\|u_{1, h}^{n}\right\|_{h}^{2}
$$

which implies

$$
\left\|u_{1, h}^{n+1}\right\|_{h}^{2} \leqslant \frac{1+\Delta t(1-\theta)}{1-\Delta t\left((1-\theta) f_{\max }^{\prime 2}+2 \theta f_{\max }^{\prime}\right)}\left\|u_{1, h}^{n}\right\|_{h}^{2},
$$

provided that $\Delta t$ satisfies (25).

Let us now consider $\mathrm{II}_{h}$

$$
u_{2, h}^{n+1}=u_{2, h}^{n}+\Delta t \beta D_{h, s(\beta)} u_{2, h}^{n+1}+\Delta t \alpha D_{2, h} u_{2, h}^{n+1} .
$$

Proceeding as before and using summation by parts we get

$$
\left(u_{2, h}^{n+1}, u_{2, h}^{n+1}\right)_{h}=\left(u_{2, h}^{n}, u_{2, h}^{n+1}\right)_{h}-\Delta t \beta\left(D_{h, s(\beta)} u_{2, h}^{n+1}, u_{2, h}^{n+1}\right)_{h}-\Delta t \alpha\left\|D_{h,-} u_{2, h}^{n+1}\right\|_{h+}^{2} .
$$

We remark that

$$
\beta\left(D_{h, s(\beta)} u_{2, h}^{n+1}, u_{2, h}^{n+1}\right)_{h} \leqslant 0 .
$$

In fact, for instance for $\beta>0$, taking $v_{h}:=u_{2, h}^{n+1}$ we have

$$
\begin{aligned}
\beta\left(D_{h,+} v_{h}, v_{h}\right)_{h} & =\beta\left(\sum_{i=2}^{N} v_{i} v_{i-1}-\sum_{i=1}^{N-1} v_{i}^{2}\right) \\
& \leqslant \beta\left(\frac{1}{2} \sum_{i=1}^{N}\left(v_{i}^{2}+v_{i-1}^{2}\right)-\sum_{i=1}^{N-1} v_{i}^{2}\right) \\
& \leqslant 0 .
\end{aligned}
$$

Taking (28) in (27) and using the discrete Friedrichs-Poincaré inequality we obtain

$$
\left\|u_{2, h}^{n+1}\right\|_{h}^{2} \leqslant \frac{1}{1+2 \alpha \Delta t(b-a)^{-2}}\left\|u_{2, h}^{n}\right\|_{h}^{2}
$$

Finally let us consider $\mathrm{III}_{h}$

$$
u_{3, h}^{n+1}=u_{3, h}^{n}+\Delta t^{2} \frac{D}{\tau} \sum_{j=1}^{n} e^{-\frac{t_{n+1}-t_{j}}{\tau}} D_{2, h} u_{h}^{j}+\Delta t^{2} \frac{D}{\tau} D_{2, h} u_{3, h}^{n+1} .
$$

As in the previous cases we get

$$
\left\|u_{3, h}^{n+1}\right\|_{h}^{2}=\left(u_{3, h}^{n}, u_{3, h}^{n+1}\right)_{h}-\Delta t^{2} \frac{D}{\tau}\left(\sum_{j=1}^{n} e^{-\frac{t_{n+1}-t_{j}}{\tau}} D_{h,-} u_{h}^{j}, D_{h,-} u_{3, h}^{n+1}\right)_{h+} \Delta t^{2} \frac{D}{\tau}\left\|D_{h,-} u_{3, h}^{n+1}\right\|_{h+}^{2} .
$$

Using the same arguments as before and due to the fact that

$$
\begin{aligned}
& 2\left(\sum_{j=1}^{n+1} e^{-\frac{t_{n+1}-t_{j}}{\tau}} D_{h,-} u_{h}^{j}, D_{h,-} u_{h}^{n+1}\right)_{h+} \\
& \quad=\left\|\sum_{j=1}^{n+1} e^{-\frac{t_{n+1}-t_{j}}{\tau}} D_{h,-} u_{h}^{j}\right\|_{h+}^{2}-e^{-2 \Delta t / \tau}\left\|\sum_{j=1}^{n} e^{-\frac{t_{n}-t_{j}}{\tau}} D_{h,-} u_{h}^{j}\right\|_{h+}^{2}+\left\|D_{h,-} u_{h}^{n+1}\right\|_{h+}^{2}
\end{aligned}
$$


we obtain

$$
\begin{aligned}
& \left\|u_{3, h}^{n+1}\right\|_{h}^{2}+\frac{D}{\tau}\left\|\Delta t\left(\sum_{j=1}^{n} e^{-\frac{t_{n+1}-t_{j}}{\tau}} D_{h,-} u_{h}^{j}+D_{h,-} u_{3, h}^{n+1}\right)\right\|_{h+}^{2} \\
& \leqslant\left\|u_{3, h}^{n}\right\|_{h}^{2}+\frac{D}{\tau}\left\|\Delta t \sum_{j=1}^{n} e^{-\frac{t_{n+1}-t_{j}}{\tau}} D_{h,-} u_{h}^{j}\right\|_{h+}^{2} .
\end{aligned}
$$

Attending that $u_{3, h}^{n}=u_{2, h}^{n+1}$ and using in (30) inequality (29) we obtain

$$
\begin{aligned}
& \left\|u_{3, h}^{n+1}\right\|_{h}^{2}+\frac{D}{\tau}\left\|\Delta t\left(\sum_{j=1}^{n} e^{-\frac{t_{n+1}-t_{j}}{\tau}} D_{h,-} u_{h}^{j}+D_{h,-} u_{3, h}^{n+1}\right)\right\|_{h+}^{2} \\
& \leqslant \frac{1}{1+2 \alpha \Delta t(b-a)^{-2}}\left\|u_{2, h}^{n}\right\|_{h}^{2}+\frac{D}{\tau}\left\|\Delta t \sum_{j=1}^{n} e^{-\frac{t_{n+1}-t_{j}}{\tau}} D_{h,-} u_{h}^{j}\right\|_{h+}^{2} .
\end{aligned}
$$

Finally, as we have $u_{2, h}^{n}=u_{1, h}^{n+1}$, from inequalities (31) and (26) we conclude the proof.

Following the proof of the last result we have the following stability result:

Theorem 4. Let $u_{h}^{j}$ and $\tilde{u}_{h}^{j}$ be solutions of the SM defined by $\mathrm{I}_{h}-\mathrm{III}_{h}$ and (17), with the conditions $u_{0}$ and $\tilde{u}_{0}$, respectively. If $\left|u_{h}^{j}\left(x_{i}\right)\right| \leqslant L,\left|\tilde{u}_{h}^{j}\left(x_{i}\right)\right| \leqslant L\left(L \in \mathbb{R}^{+}\right)$, for $i=1, \ldots, N$ and $j=1, \ldots, M, f$ is continuously differentiable and $f(0)=0$, then

$$
E_{h}\left(u_{h}^{n+1}-\tilde{u}_{h}^{n+1}\right) \leqslant(S(\Delta t, \theta))^{n+1}\left\|u_{0}-\tilde{u}_{0}\right\|_{h}^{2},
$$

for $n=0, \ldots, M-1$, where the stability factor $S(\Delta t, \theta)$ is defined by (24) and provided that the time stepsize satisfies (25).

Following the arguments used in the proof of Theorem 3, we may obtain an estimate for the discrete energy (22) of the solution of the NSM defined by (18) and (17) when homogeneous boundary conditions are considered. A stability result can be also established when the initial condition is perturbed, more precisely we have:

Theorem 5. Let $u_{h}^{j}, \tilde{u}_{h}^{j}$ be solutions of the NSM defined by (18) and (17), with initial conditions $u_{0}$ and $\tilde{u}_{0}$, respectively. If $\left|u_{h}^{j}\left(x_{i}\right)\right| \leqslant L,\left|\tilde{u}_{h}^{j}\left(x_{i}\right)\right| \leqslant L\left(L \in \mathbb{R}^{+}\right)$, for $i=1, \ldots, N$ and $j=1, \ldots, M, f$ is continuously differentiable and $f(0)=0$, then

$$
E_{h}\left(u_{h}^{n+1}-\tilde{u}_{h}^{n+1}\right) \leqslant(S(\Delta t, \theta))^{n+1}\left\|u_{0}-\tilde{u}_{0}\right\|_{h}^{2},
$$

for $n=0, \ldots, M-1$, where the stability factor $S(\Delta t, \theta)$ is defined by

$$
S(\Delta t, \theta)=\frac{1+\Delta t(1-\theta)}{\min \left\{1,1-\Delta t\left((1-\theta) f_{\max }^{\prime 2}+2 \theta f_{\max }^{\prime}-(2 \alpha+D / \tau \Delta t)(b-a)^{-2}\right)\right\}}
$$

for $\theta \in[0,1]$ and $f_{\max }^{\prime}=\max _{|u| \leqslant L} f^{\prime}(u)$, provided that

$$
1-\Delta t\left((1-\theta) f_{\max }^{\prime 2}+2 \theta f_{\max }^{\prime}-(2 \alpha+D / \tau \Delta t)(b-a)^{-2}\right)>0 .
$$

The stability restriction for the time stepsize defined by (35) is stronger than the restriction (25) for the SM in the sense that the first one implies a smaller time stepsize than the second one.

We denote by SI the splitting method $\mathrm{I}_{h}-\mathrm{III}_{h}$ with $\theta=1$ (implicit reaction), SE the splitting method $\mathrm{I}_{h}-\mathrm{III}_{h}$ with $\theta=0$ (explicit reaction), by FI the non-splitting scheme (18) with $\theta=1$ (implicit reaction) and by IMEX the nonsplitting scheme (18) with $\theta=0$ (explicit reaction) and by $S_{i}, S_{e}, S_{f i}$ and $S_{i m e x}$ we represent the corresponding stability factors. 


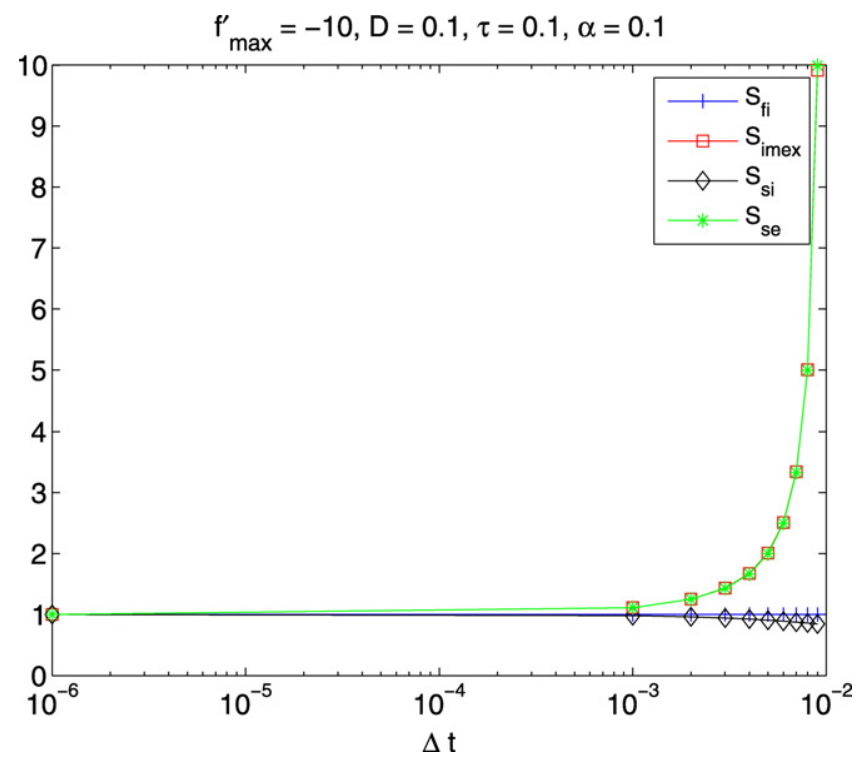

Fig. 1. Stability factor: $f_{\max }^{\prime}<0$.

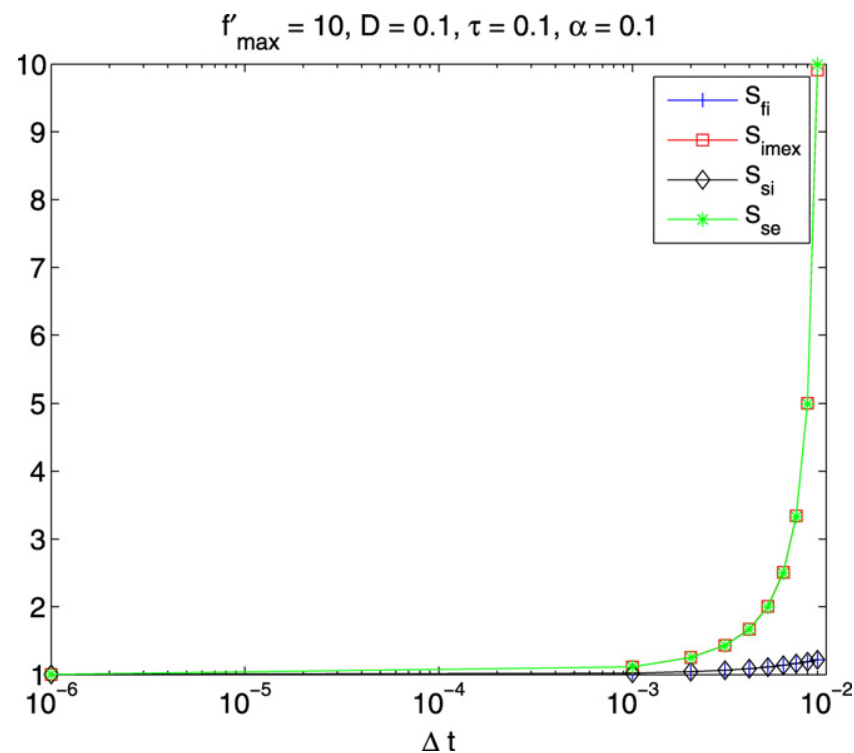

Fig. 2. Stability factor: $f_{\max }^{\prime}>0$.

In Figs. 1-2 we plot the defined stability factors as functions of the time step. As we expected, in what concerns the stability, these figures confirm the advantage of the implicit schemes. If we compare the splitting schemes with the non-splitting ones we may see, specially for $f_{\max }^{\prime}<0$ (Fig. 1), that the stability factor for the splitting method with implicit reaction is less or equal than the stability factor of the non-splitting scheme with implicit reaction.

The behavior of the stability conditions to the time step is considered in Fig. 3. Let $S C(\Delta t, \theta)$ be defined by

$$
S C(\Delta t, \theta)=\max \left\{0,1-\Delta t\left((1-\theta) f_{\max }^{\prime 2}+2 \theta f_{\max }^{\prime}\right)\right\},
$$

for splitting schemes and by

$$
S C(\Delta t, \theta)=\max \left\{0,1-\Delta t\left((1-\theta) f_{\max }^{\prime 2}+2 \theta f_{\max }^{\prime}-(2 \alpha+D / \tau \Delta t)(b-a)^{-2}\right)\right\},
$$




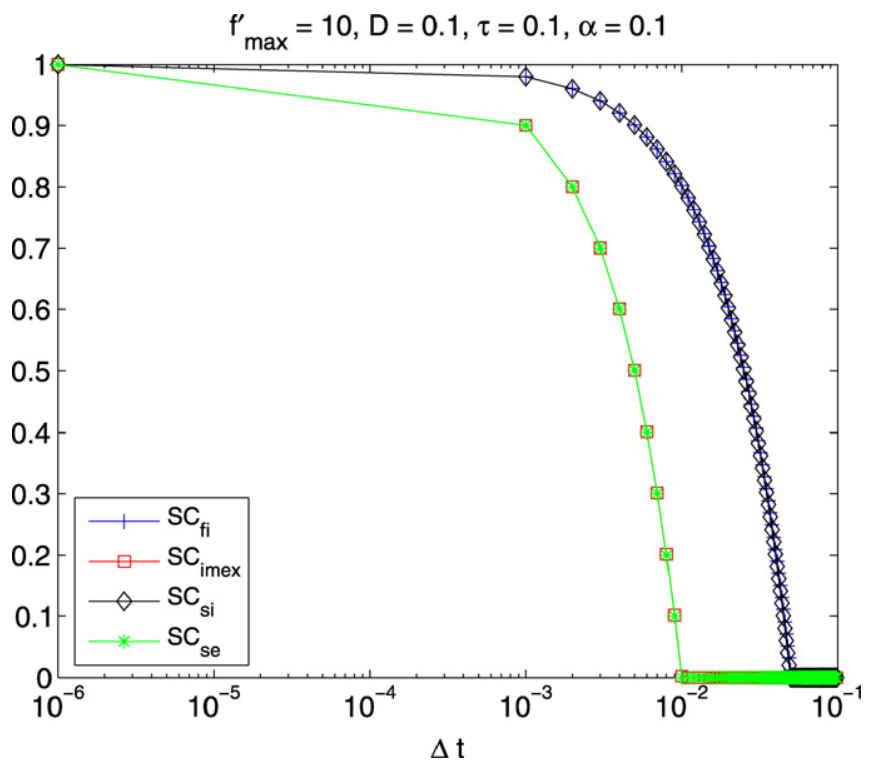

Fig. 3. Stability conditions.

for non-splitting ones. By $S C_{i}, S C_{e}, S C_{f i}$ and $S C_{i m e x}$ we denote the previous functions. Fig. 3 illustrates the fact that the restrictions to the stability imposed by the explicit schemes are more restrictive.

The spitting method - SM - and the non-splitting method - NSM - studied in this paper were defined replacing the first-order partial derivative with respect to space variable by the first-order backward finite difference operator $D_{h,-}$ or first-order forward finite difference operator $D_{h,+}$ depending on the signal of $\beta$. Let $D_{c}$ be the second-order centered finite difference operator defined by

$$
D_{c} v_{h}\left(x_{i}\right)=\frac{v_{h}\left(x_{i+1}\right)-v_{h}\left(x_{i-1}\right)}{2 h} .
$$

Attending that for $v_{h}$ such that $v_{h}\left(x_{0}\right)=v_{h}\left(x_{N}\right)=0$ we have

$$
\left(D_{c} v_{h}, v_{h}\right)_{h}=0
$$

we conclude that for the SM and the NSM defined replacing $\frac{\partial u}{\partial x}\left(x_{i}, t_{n}\right)$ by $D_{c} u_{h}^{n}\left(x_{i}\right)$ hold stability results analogous to Theorem 4 for the SM and to Theorem 5 for the NSM.

\subsection{Error estimates}

In this section we will study the convergence of the numerical schemes proposed in the previous section. Let $e_{h}^{j}\left(x_{i}\right)=u_{h}^{j}\left(x_{i}\right)-u\left(x_{i}, t_{j}\right)$ be the global error of the approximation $u_{h}^{j}\left(x_{i}\right)$ obtained by the numerical method $\mathrm{I}_{h}-\mathrm{III}_{h}$ with boundary conditions (17), and let $T_{h}^{j}\left(x_{i}\right)$ be the corresponding truncation error. Following the proof of Theorem 3 we may prove the next result.

Theorem 6. Let $u_{h}^{j}, j=1, \ldots, M$, be the numerical solution of (10)-(11) obtained with $\mathrm{I}_{h}-\mathrm{III}_{h}$ with boundary conditions (17). If $f$ is continuously differentiable and $f(0)=0$, then

$$
E_{h}\left(e_{h}^{n+1}\right) \leqslant \sum_{j=0}^{n} \bar{S}^{j+1}(\Delta t, \theta) \Delta t\left\|T_{h}^{n+1-j}\right\|_{h}^{2},
$$

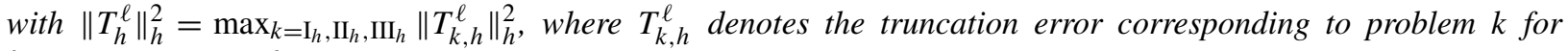
$k=\mathrm{I}_{h}, \mathrm{II}_{h}, \mathrm{III}_{h}$, and

$$
\bar{S}(\Delta t, \theta)=\frac{M(\Delta t, \theta)}{m(\Delta t, \theta)},
$$


where

$$
M(\Delta t, \theta)=\max \left\{e^{-2 \Delta t / \tau}, \frac{1+\Delta t(1-\theta)}{1-\Delta t\left((1-\theta) f_{\max }^{\prime 2}+2 \theta f_{\max }^{\prime}+1\right)}\right\}
$$

and

$$
m(\Delta t, \theta)=\min \left\{1,1-\Delta t\left(1-\frac{D}{\tau} \Delta t(b-a)^{-2}\right)\right\},
$$

provided that

$$
1-\Delta t\left((1-\theta) f_{\max }^{\prime 2}+2 \theta f_{\max }^{\prime}+1\right)>0
$$

and

$$
1-\Delta t\left(1-\frac{D}{\tau} \Delta t(b-a)^{-2}\right)>0 .
$$

Proof. Let

$$
e_{k, h}^{j}\left(x_{i}\right)=u_{k, h}^{j}\left(x_{i}\right)-u_{k}\left(x_{i}, t_{j}\right), \quad k=1,2,3,
$$

be the error for the different subproblems $\mathrm{I}_{h}-\mathrm{III}_{h}$. Let us first consider $\mathrm{I}_{h}$. Considering the error equation for $e_{1, h}^{n+1}$ it can be shown that

$$
\left\|e_{1, h}^{n+1}\right\|_{h}^{2} \leqslant \frac{(1+\Delta t(1-\theta))\left\|e_{1, h}^{n}\right\|_{h}^{2}+\Delta t\left\|T_{1, h}^{n+1}\right\|_{h}^{2}}{1-\Delta t\left((1-\theta) f_{\max }^{\prime 2}+2 \theta f_{\max }^{\prime}+1\right)},
$$

provided that (36) holds.

Let us now consider $\mathrm{II}_{h}$. Using on the error equation for $e_{2, h}^{n+1}$ the arguments considered on the proof of inequality (29), it can be shown that

$$
\left\|e_{2, h}^{n+1}\right\|_{h}^{2}=\left\|e_{2, h}^{n}\right\|_{h}^{2}-2 \alpha(b-a)^{-2}\left\|D_{h,-} e_{2, h}^{n+1}\right\|_{h+}^{2}+2 \Delta t\left(T_{2, h}^{n+1}, e_{2, h}^{n+1}\right)_{h} .
$$

Attending that

$$
\left(T_{2, h}^{n+1}, e_{2, h}^{n+1}\right)_{h} \leqslant \frac{1}{\epsilon^{2}}\left\|T_{2, h}^{n+1}\right\|_{h}^{2}+\epsilon^{2}\left\|e_{2, h}^{n+1}\right\|_{h}^{2}
$$

holds for $\epsilon \neq 0$, considering $\epsilon^{2}=2 \alpha(b-a)^{-2}$, we obtain

$$
\left\|e_{2, h}^{n+1}\right\|_{h}^{2} \leqslant\left\|e_{2, h}^{n}\right\|_{h}^{2}+\frac{\Delta t}{2 \alpha \Delta t(b-a)^{-2}}\left\|T_{2, h}^{n+1}\right\|_{h}^{2} .
$$

Finally let us consider $\mathrm{III}_{h}$. From the error equation for $e_{3, h}^{n+1}$, considering the procedures used on the proof of inequality (30), it can be shown

$$
\begin{gathered}
m(\Delta t, \theta)\left(\left\|e_{3, h}^{n+1}\right\|_{h}^{2}+\frac{D}{\tau}\left\|\Delta t\left(\sum_{j=1}^{n} e^{-\frac{t_{n+1}-t_{j}}{\tau}} D_{h,-} e_{h}^{j}+D_{h,-} e_{3, h}^{n+1}\right)\right\|_{h+}^{2}\right) \\
\leqslant\left\|e_{3, h}^{n}\right\|_{h}^{2}+\frac{D}{\tau} e^{-2 \Delta t / \tau}\left\|\Delta t \sum_{j=1}^{n} e^{-\frac{t_{n+1}-t_{j}}{\tau}} D_{h,-} e_{h}^{j}\right\|_{h+}^{2}+\Delta t\left\|T_{3, h}^{n+1}\right\|_{h}^{2} .
\end{gathered}
$$

Combining (38)-(40) and attending that $e_{1, h}^{n}=e_{h}^{n}, e_{2, h}^{n}=e_{1, h}^{n+1}$ and $e_{3, h}^{n}=e_{2, h}^{n+1}$, we conclude that

$$
E_{h}\left(e_{h}^{n+1}\right) \leqslant \bar{S}(\Delta t, \theta) E_{h}\left(e_{h}^{n}\right) .
$$

As $E_{h}\left(e_{h}^{0}\right)=0$, we conclude the proof.

According to Theorem 6, we conclude that, if

$$
M(\Delta t, \theta)=e^{-2 \Delta t / \tau},
$$


we have

$$
E_{h}\left(e_{h}^{n+1}\right) \leqslant e^{C T}\left\|T_{h}\right\|_{h, \infty}^{2},
$$

where $C=-2 / \tau$ if $m(\Delta t, \theta)=1$, and

$$
C=\frac{1}{1-\Delta t\left(1-\frac{D}{\tau} \Delta t(b-a)^{-2}\right)}
$$

if

$$
m(\Delta t, \theta)=1-\Delta t\left(1-\frac{D}{\tau} \Delta t(b-a)^{-2}\right) .
$$

For

$$
M(\Delta t, \theta)=\frac{1+\Delta t(1-\theta)}{1-\Delta t\left((1-\theta) f_{\max }^{\prime 2}+2 \theta f_{\max }^{\prime}+1\right)}
$$

we may obtain similar results.

The convergence of the $\theta$ family of methods is now consequence of the consistency, that is, $\left\|T_{h}\right\|_{h, \infty}=\mathcal{O}(h, \Delta t)$.

\section{Numerical results}

Our aim in this section is to illustrate the theoretical results established in the last section namely the stability results. In all numerical examples we consider the convection-diffusion-reaction equation

$$
\frac{\partial u}{\partial t}(x, t)=f(u)+\beta \frac{\partial u}{\partial x}(x, t)+\alpha \frac{\partial^{2} u}{\partial x^{2}}(x, t)+\frac{D}{\tau} \int_{0}^{t} e^{-\frac{t-s}{\tau}} \frac{\partial^{2} u}{\partial x^{2}}(x, s) \mathrm{d} s, \quad x \in(0,50), t \in(0, T] .
$$

Example 1. Let us consider (41) with

$$
f(u)=U u(1-u),
$$

and $U=10, \alpha=0.1, D=0.1, \tau=0.01, \beta=-0.5$ and $T=5$. Eq. (41) is complemented with initial and boundary conditions

$$
\begin{aligned}
& u(x, 0)= \begin{cases}1, & x \in[0,5], \\
0, & x \in(5,50],\end{cases} \\
& u(0, t)=1, \quad u(50, t)=0, \quad t \in(0,5] .
\end{aligned}
$$

In Fig. 4 we plot the numerical solution computed with the method FI defined by (18) $(\theta=1)$, with $\Delta t=0.001$ and $h=0.1$. This solution is adopted as reference solution in what follows. We remark that the same results were obtained with the methods IMEX, SE and SI.

Let us consider now only the implicit methods FI and SI. In Figs. 5 and 6 we plot the numerical solutions obtained with $\Delta t=0.01$.

From Figs. 5 and 6 we observe that an increasing of the time stepsize implies in the method FI an erratic behavior: the numerical traveling wave solution propagates with a higher speed than the corresponding solutions obtained with the splitting method SI.

When the method SI is used, we solve, in each time step, a diagonal non-linear system. Otherwise, when we use the method FI, we solve, in each time step, a tridiagonal non-linear system. Attending to this facts the first method is cheaper than the second one. Such remark can be concluded from the CPU time of both methods. We observed in all numerical experiments the following relation between the CPU times of both methods:

$$
\frac{C P U(F I)}{C P U(S I)} \simeq 1.85 \text {. }
$$

Let us compare now the splitting methods. We consider $U=-20, \alpha=0.1, D=0.1, \tau=0.1, \beta=0, T=2$ and the conditions (42). In Fig. 7 we plot the numerical solutions obtained using the method SE for several values of $\Delta t$. 

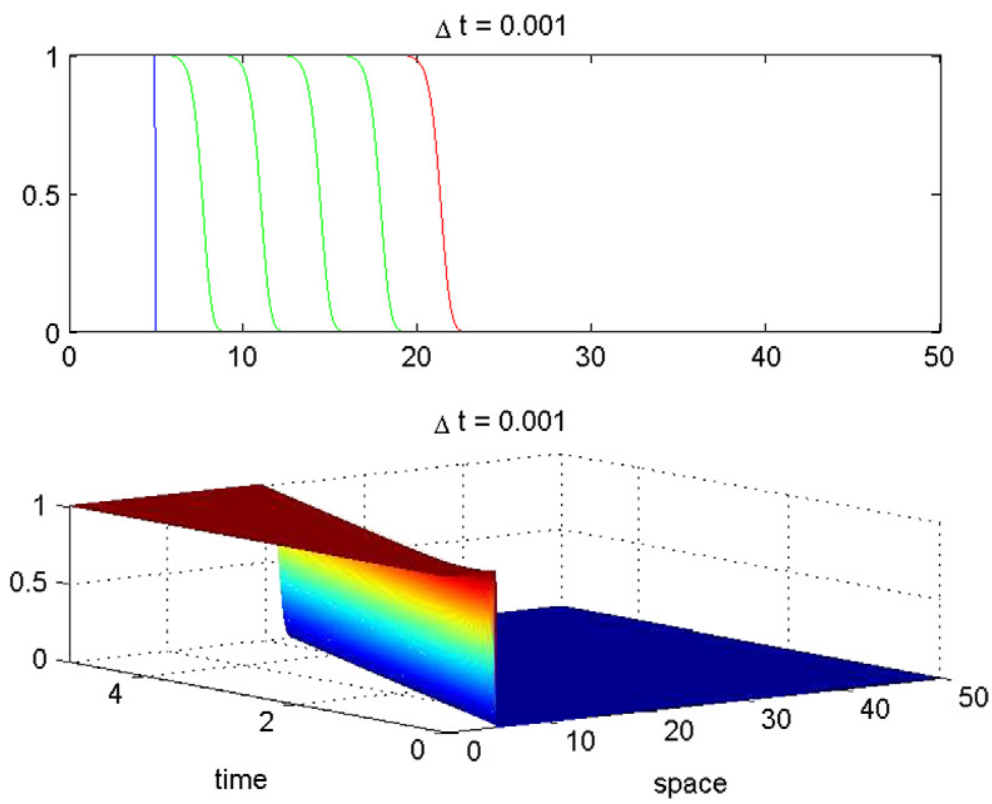

Fig. 4. The reference solution computed with the method FI.
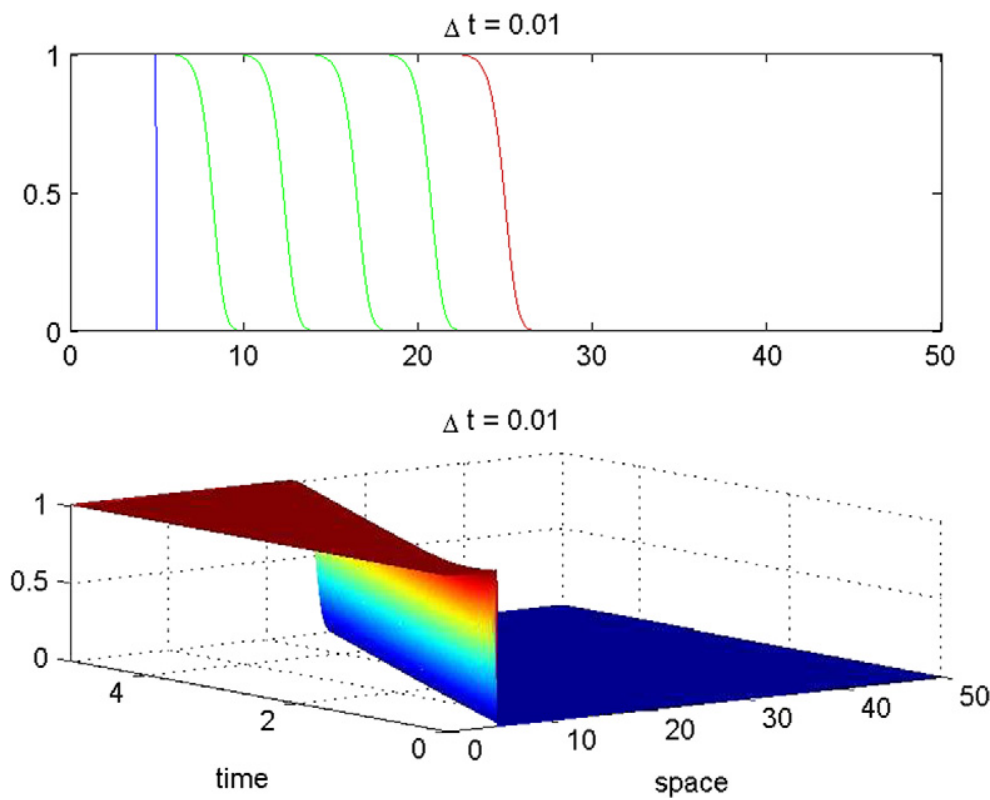

Fig. 5. Numerical solutions computed using the non-splitting method FI.

We observe that when $\Delta t$ increases the numerical solution presents an instable behavior. As the stability restriction of the implicit method SI is weaker than the corresponding restriction of the explicit one, the behavior presented in Fig. 7 is not observed when the method SI is considered. In Fig. 8 we plot only the numerical solution obtained with the method SI for $\Delta t=0.1$.

In the following example we analyze the sharpness of the restriction (25) to the time stepsize which allowed the establishment of the stability result for the splitting methods: Theorem 4. 

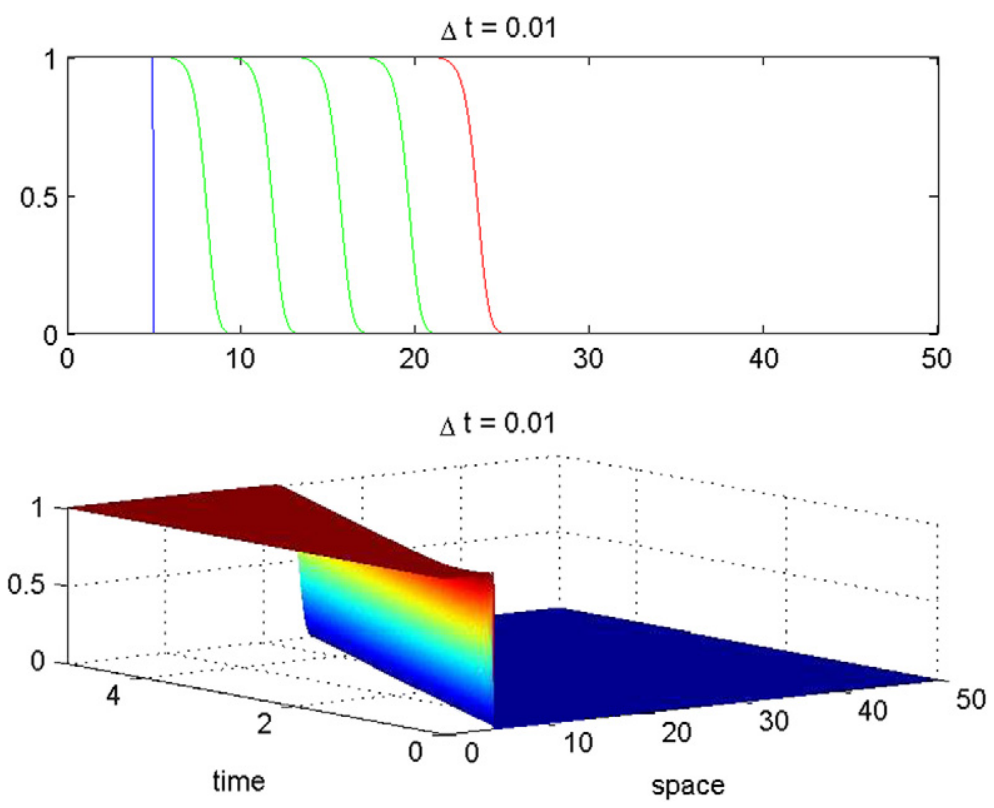

Fig. 6. Numerical solutions computed using the splitting method SI.
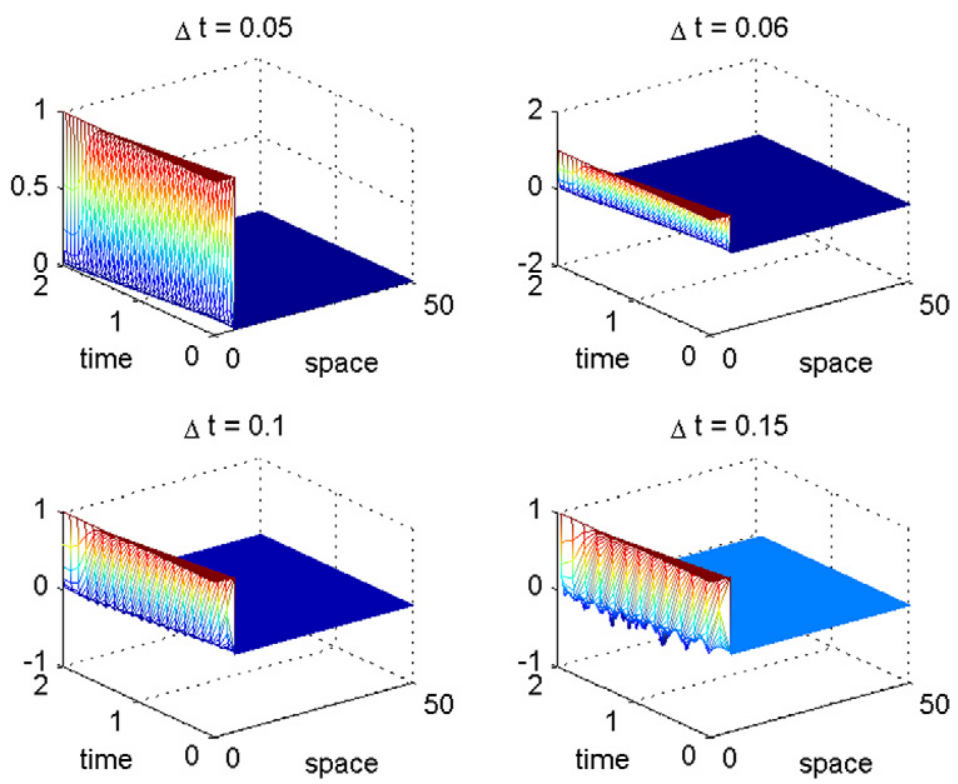

Fig. 7. Numerical solutions computed using the method SE.

Example 2. Let us consider the IBVP defined by Eq. (41) with

$$
f(u)=-2 u^{2},
$$

by Dirichlet homogeneous boundary conditions and by the initial condition

$$
u(x, 0)=8.1 \operatorname{sech}(x-25)^{2}, \quad x \in(0,50) .
$$

In Eq. (41) we take $\alpha=0.1, D=0.1, \tau=0.1$ and $\beta=0$.

In Figs. 9 and 10 we plot the numerical results obtained using the methods SE and SI respectively, when $\Delta t$ increases. 


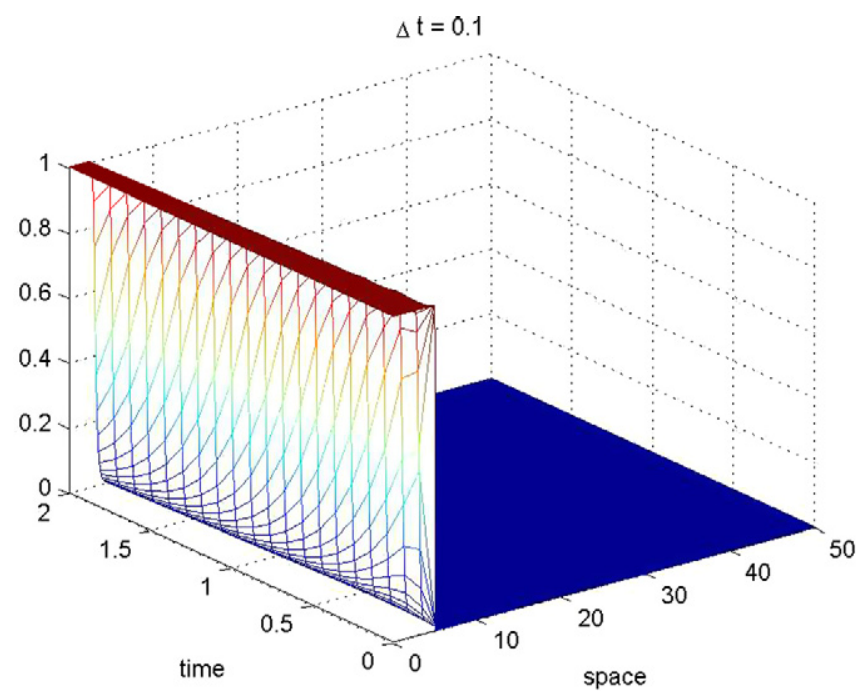

Fig. 8. Numerical solutions computed using the method SI.
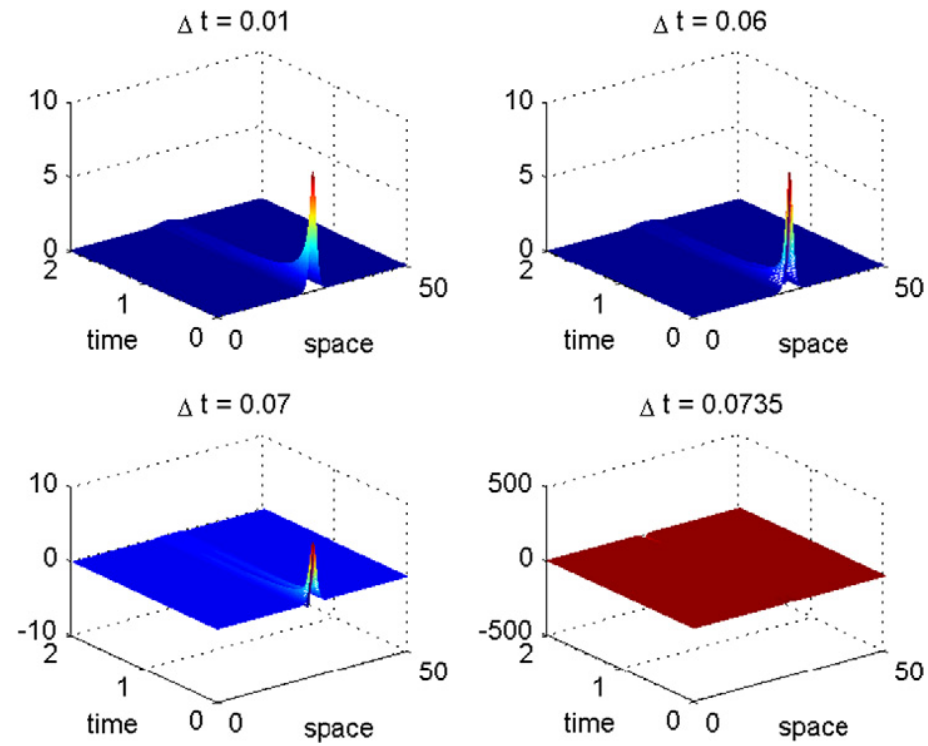

Fig. 9. Numerical solutions computed using the method SE.

Based on the numerical experiments presented in Fig. 9 we conclude that the upper stability bound $\Delta t_{0}$ for the stepsize is in $[0.06,0.07)$. However, if we use the condition (25) we conclude that $\Delta t_{0} \leqslant 1 / f_{\max }^{\prime 2} \simeq 0.00381$. We remark that the upper bound deduced from the numerical results is approximately equal to $1 /\left|f_{\max }^{\prime}\right|$. This conclusion shows that the theoretical restriction (25) is sharp in the present context.

The last example shows that the condition (25) could be sharp. Nevertheless we point out that this condition can be replaced by

$$
1-\Delta t\left((1-\theta)\left|f_{\max }^{\prime}\right|+2 \theta f_{\max }^{\prime}\right)>0 .
$$

In this case the definition (24) of $S(\Delta t, \theta)$ should be replaced by the following

$$
S(\Delta t, \theta)=\frac{1+\Delta t(1-\theta)\left|f_{\max }^{\prime}\right|}{\left(1+2 \alpha \Delta t(b-a)^{-2}\right)\left(1-\Delta t\left((1-\theta)\left|f_{\max }^{\prime}\right|+2 \theta f_{\max }^{\prime}\right)\right)} .
$$



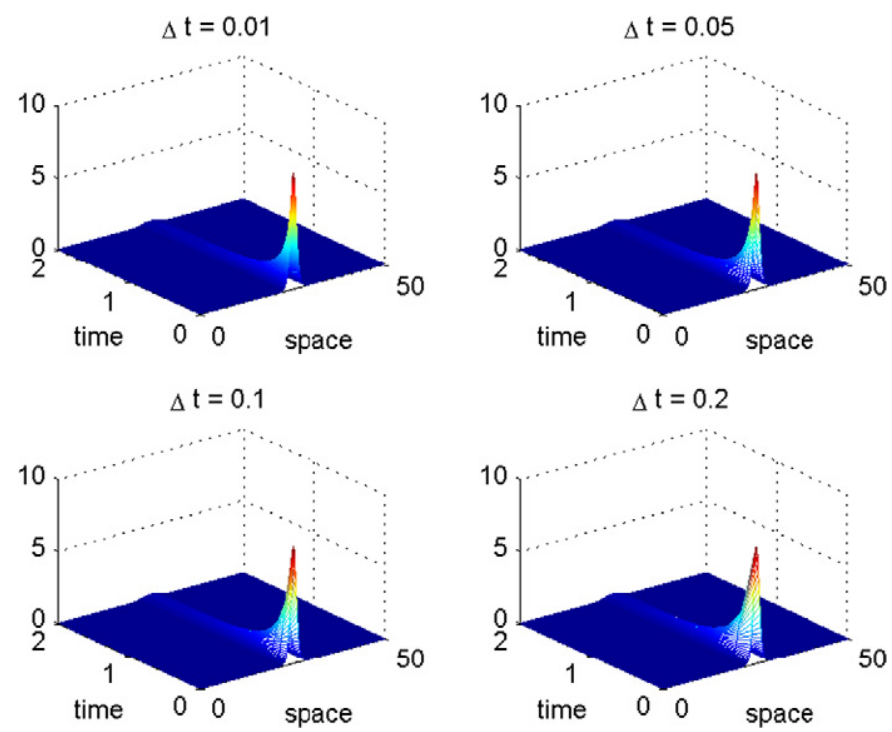

Fig. 10. Numerical solutions computed using the method SI.

This means that Theorem 4 holds with $S(\Delta t, \theta)$ given by (44) under the new condition (43) for the time stepsize.

\section{References}

[1] A. Araújo, J.A. Ferreira, P. de Oliveira, Qualitative behaviour of numerical traveling waves solutions for reaction diffusion equations with memory, Applicable Analysis 84 (2005) 1231-1246.

[2] A. Araújo, J.A. Ferreira, P. de Oliveira, The effect of memory terms in diffusion phenomena, Journal of Computational Mathematics 24 (2006) 91-102.

[3] S. Barbeiro, J.A. Ferreira, Integro-differential models for percutaneous drug absortion, International Journal of Computer Mathematics 84 (2007) 451-467.

[4] J.R. Branco, J.A. Ferreira, P. de Oliveira, Numerical methods for the generalized Fisher-Kolmogorov-Petrovskii-Piskunov equation, Applied Numerical Mathematics 57 (2007) 89-102.

[5] C. Cattaneo, Sulla condizione de calore, Atti del Seminario Matematico e Fisico dell' Universitá de Modena 3 (1948) 3-21.

[6] S. Fedotov, Traveling waves in a reaction-diffusion system: diffusion with finite velocity and Kolmogorov-Petrovskii-Piskunov kinetics, Physical Review E 4 (1998) 5143-5145.

[7] S. Fedotov, Nonuniform reaction rate distribution for the generalized Fisher equation: ignition ahead of the reaction front, Physical Review E 4 (1998) 4958-4961.

[8] S. Fedotov, Y. Okuda, Non Markovian random process and traveling front in a reaction transport system with memory and long-range interactions, Physical Review E 66 (2002) 021113 (7 pages).

[9] S. Fedotov, Y. Okuda, Waves in a reaction-transport system with memory, long-range interactions and transmutations, Physical Review E 70 (2004) 051108, (10 pages).

[10] J.A. Ferreira, P. de Oliveira, Memory effects and random walks in reaction-transport systems, Applicable Analysis 86 (2007) 99-118.

[11] D.D. Joseph, L. Preziosi, Heat waves, Reviews of Modern Physics 61 (1989) 41-73.

[12] P. Vernotte, La véritable de équation de la chaleur, Comptes Rendus de l'Académie des Sciences 247 (1958). 